\title{
In Situ Syntheis of Metal Nanoparticles and Selective Naked- Eye Detection of Lead Ions from Aqueous Media
}

\author{
K. Yoosaf, Binil Itty Ipe and K. George Thomas*
}

Photosciences and Photonics, Regional Research Laboratory (CSIR), Trivandrum 695 019, India

\section{Table of Content}

1. Expansions of abbreviated references $(8 \& 29)$

2. Instrumental methods and spectroscopic investigations

3. Absorption spectra of gold nanoparticles after adding 4-hydroxy benzoic acid and pyrogallol

4. Absorption spectra of gallic acid and Au nanoparticles

5. FTIR spectra of gallic acid and Au nanoparticles

6. Absorption spectra of $\mathrm{Au}$ nanoparticles in presence of different metal ions at $\mathrm{pH} 3.0$

7. Absorption spectra of $\mathrm{Au}$ nanoparticles in presence of different metal ions at $\mathrm{pH} 4.5$

8. Absorption spectra of Ag nanoparticles in presence of different metal ions at $\mathrm{pH} 4.5$

9. Effect of EDTA

10. Absorption spectra of gold nanoparticles in presence of $\mathrm{Pb}^{2+}$ ion at $\mathrm{pH} 6.0$

11. Sensitivity of $\mathrm{Au}$ nanoparticles towards $\mathrm{Pb}^{2+}$ ions in presence of other interfering metal cations

12. Model Calculations
Page No.

S2

S2

S3

S4

S4

S5

S6

S7

S8

S8

S9

S9 


\section{Expansions of abbreviated references 8 and 29}

8. (b) Brennan, J. L.; Hatzakis, N. S.; Tshikhudo, T. R.; Dirvianskyite, N.; Razumas, V.; Patkar, S.;

Vind, J.; Svendsen, A.; Nolte, R. J. M.; Rowan, A. E.; Brust, M. Bioconjugate Chem. 2006, 17, 1373-1375.

29. Gaussian 03, Version 6.1, Frisch, M. J.; Trucks, G. W.; Schlegel, H. B.; Scuseria, G. E.; Robb, M. A.; Cheeseman, J. R.; Montgomery, Jr. J. A.; Vreven, T.; Kudin, K. N.; Burant, J. C.; Millam, J. M.; Iyengar, S. S.; Tomasi, J.; Barone, V.; Mennucci, B.; Cossi, M.; Scalmani, G.; Rega, N.; Petersson, G. A.; Nakatsuji, H.; Hada, M.; Ehara, M.; Toyota, K.; Fukuda, R.; Hasegawa, J.; Ishida, M.; Nakajima, T.; Honda, Y.; Kitao, O.; Nakai, H.; Klene, M.; Li, X.; Knox, J. E.; Hratchian,H. P.; Cross,J. B.; Adamo, C.; Jaramillo, J.; Gomperts, R.; Stratmann, R. E.; Yazyev, O.; Austin, A. J.; Cammi, R.; Pomelli, C.; Ochterski, J. W.; Ayala, P. Y.; Morokuma, K.; Voth, G. A.; Salvador, P.; Dannenberg, J. J.; Zakrzewski,V. G.; Dapprich, S.; Daniels, A. D.; Strain, M. C.; Farkas, O.; Malick, D. K.; Rabuck, A. D.; Raghavachari, K.; Foresman, J. B.; Ortiz, J. V.; Cui, Q.; Baboul, A. G.; Clifford, S.; Cioslowski, J.; Stefanov, B. B.; Liu, G.; Liashenko, A.; Piskorz, P. ; Komaromi, I.; Martin, R. L.; Fox, D. J.; Keith, T.; Al-Laham, M. A.; Peng, C. Y.; Nanayakkara, A.; Challacombe, M.; Gill, P. M. W.; Johnson, B.; Chen, W.; Wong, M. W.; Gonzalez, C.; Pople, J. A., Gaussian, Inc., Pittsburgh PA, 2003.

\section{Instrumental methods and spectroscopic investigations}

Analytical grade $\left[\mathrm{HAuCl}_{4}\right] \cdot 3 \mathrm{H}_{2} \mathrm{O}$ was purchased from Aldrich and used as received. Synthetic grade Gallic acid was purchased from s.d. fine chemicals and recrystallized from a saturated solution of methanol. Silver nitrate, Lead(II) acetate, Nickel(II) acetate, Magnesium(II) sulphate, Copper(II) sulphate, Zinc(II) sulphate, Calcium(II) chloride, Cadmium(II) bromide, Iron(III) chloride, EDTA and sodium hydroxide were obtained from s.d. fine chemicals and used as received. Stock solutions of all the reagents were prepared in doubly distilled water. 
All the stock solutions were prepared by dissolving the desired compound in doubly distilled water. IR spectra of aqueous solution were recorded on a Nicolet Impact 400D infrared spectrophotometer using ATR technique. The electronic absorption spectra were recorded on a Shimadzu Model UV-3101 PC UV-Vis-NIR scanning spectrophotometer. For TEM studies, a drop of colloidal gold solution was placed on a carbon coated $\mathrm{Cu}$ grid and the solvent was allowed to evaporate. Specimens were examined on a Hitachi H600 transmission electron microscope. AFM measurements were carried out by drop casting the solution on a freshly cleaved mica surface. Specimens were imaged on a multimode scanning probe microscope (Nanoscope IV controller, digital instruments) using the tapping mode technique. $\mathrm{pH}$ of the solution was determined using Elico LI 612 $\mathrm{pH}$ analyser. The zeta potential of nanoparticles at different $\mathrm{pH}$ values were recorded using Malvern Zetasizer 3000HSA.

\section{Absorption spectra of gold nanoparticles after adding 4-hydroxy benzoic acid and pyrogallol}

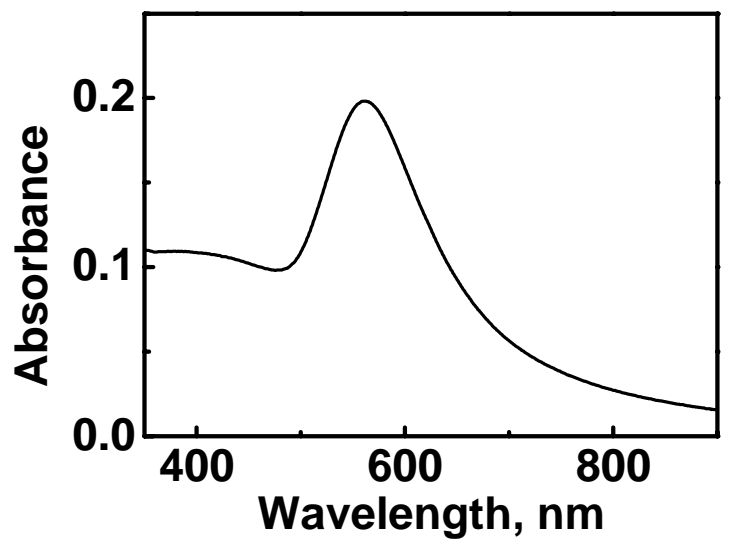

Figure S3. Absorption spectrum of gold nanoparticles prepared by adding a 1:3 mixture of 4-hydroxy benzoic acid and pyrogallol to $\mathrm{HAuCl}_{4}$ solution. 
4. Absorption spectra of gallic acid and gold nanoparticles

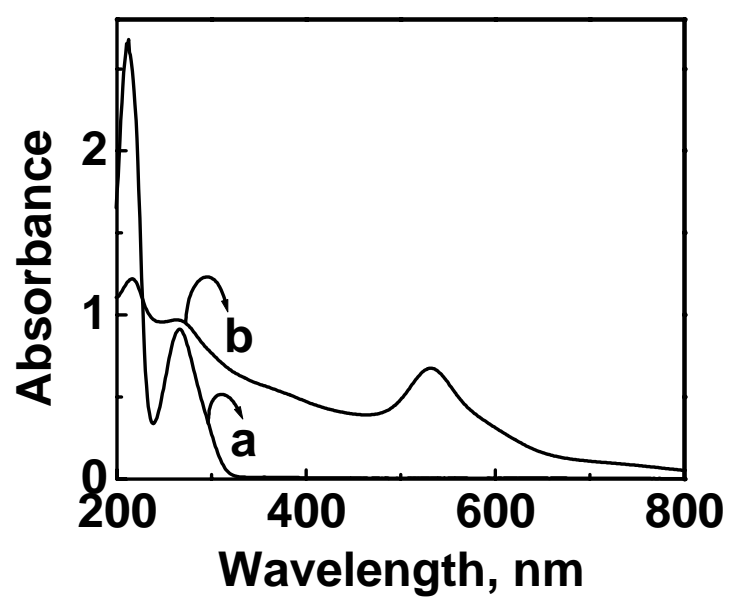

Figure S4. Absorption spectra of gallic acid (trace a) and gold nanoparticles (trace b).

5. FTIR spectra of gallic acid and gold nanoparticles

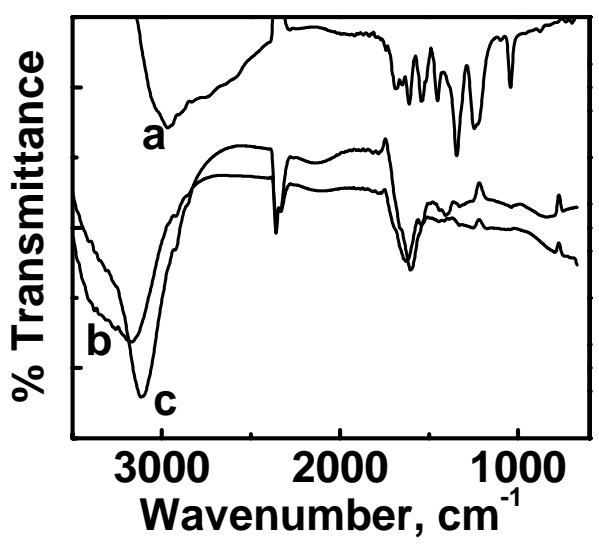

Figure S5. FTIR spectra of gallic acid (trace a), gold nanoparticles (trace b) and gold nanoparticles after the addition of $75 \mu \mathrm{M} \mathrm{Pb}^{2+}$ (trace $\mathrm{c}$ ). 
6. Absorption spectra of gold nanoparticles in presence of $\mathrm{Pb}^{2+}$ ion at $\mathrm{pH} 3.0$
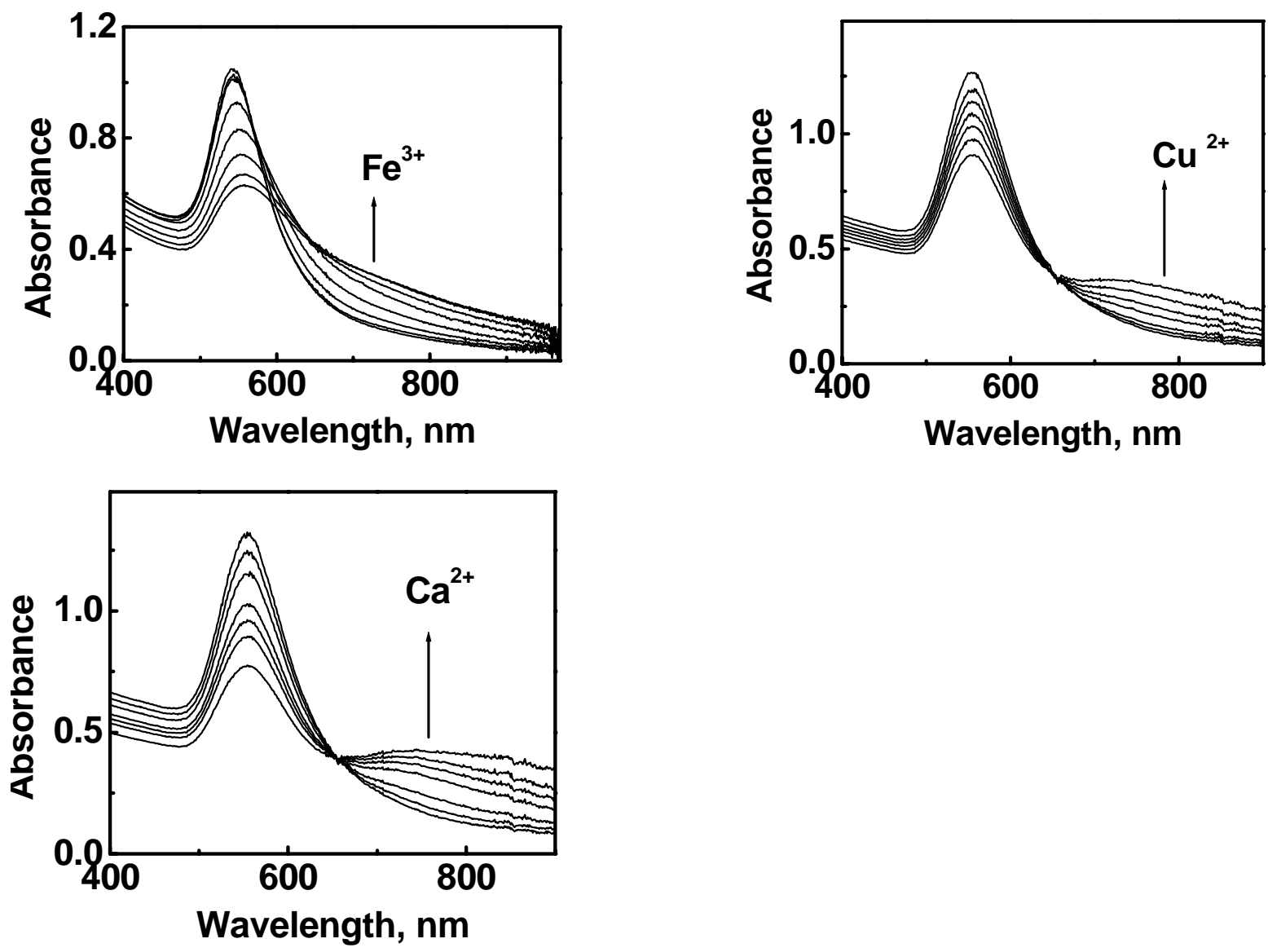

Figure S6. Absorption spectral changes of gold nanoparticles in water ( $\mathrm{pH} 3.0)$ on addition of 0-100 $\mu \mathrm{M} \mathrm{Pb}^{2+}$ (Note: Solution undergoes rapid precipitation) Absorption spectra of gallic acid stabilized gold nanoparticles in presence of various amounts of $\mathrm{Fe}^{3+}, 20,30,40,50 \mu \mathrm{M}$ ferric chloride solution 
7. Absorption spectra of gold nanoparticles in presence of different metal ions at $\mathbf{p H} 4.5$
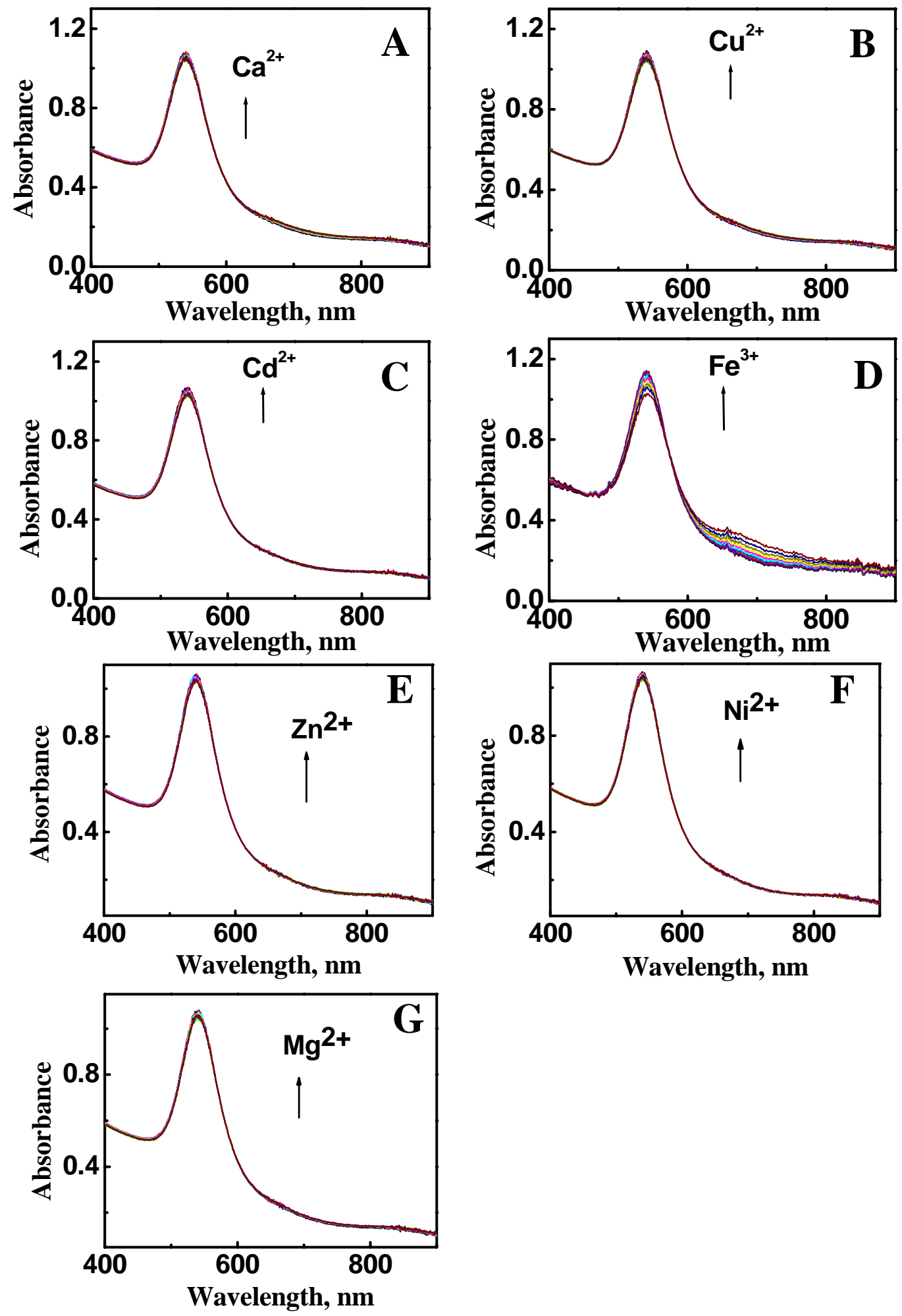

Figure S7. Absorption spectral changes of gold nanoparticles in water on addition of (A) $\mathrm{Ca}^{2+}$,

$$
\mathrm{Cu}^{2+}, \text { (C) } \mathrm{Cd}^{2+}, \text { (D) } \mathrm{Fe}^{3+}, \text { (E) } \mathrm{Zn}^{2+},(\mathrm{F}) \mathrm{Ni}^{2+} \text { and }(\mathrm{G}) \mathrm{Mg}^{2+} \text { ions at } 0-100 \mu \mathrm{M} \text { at } \mathrm{pH} 4.5
$$


8. Absorption spectra of silver nanoparticles in presence of different metal ions at pH 4.5
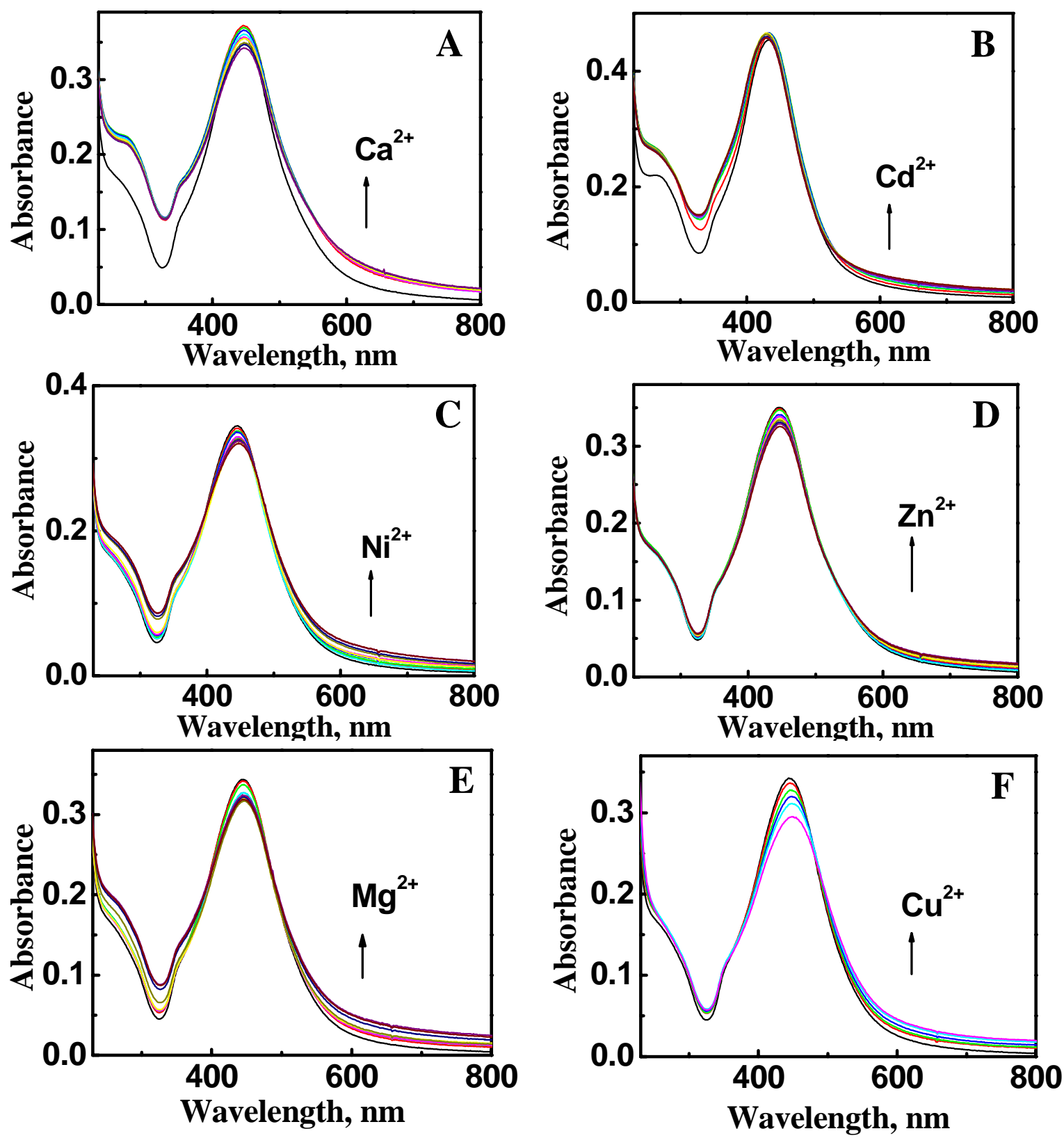

Figure S8. Absorption spectral changes of silver nanoparticles in water on addition of (A) $\mathrm{Ca}^{2+}$, (B) $\mathrm{Cd}^{2+}$, (C) $\mathrm{Ni}^{2+}$, (D) $\mathrm{Zn}^{2+}$, (E) $\mathrm{Mg}^{2+}$ and (F) $\mathrm{Cu}^{2+}$ at $0-100 \mu \mathrm{M}$ at $\mathrm{pH} 4.5$. 


\section{Effect of EDTA}

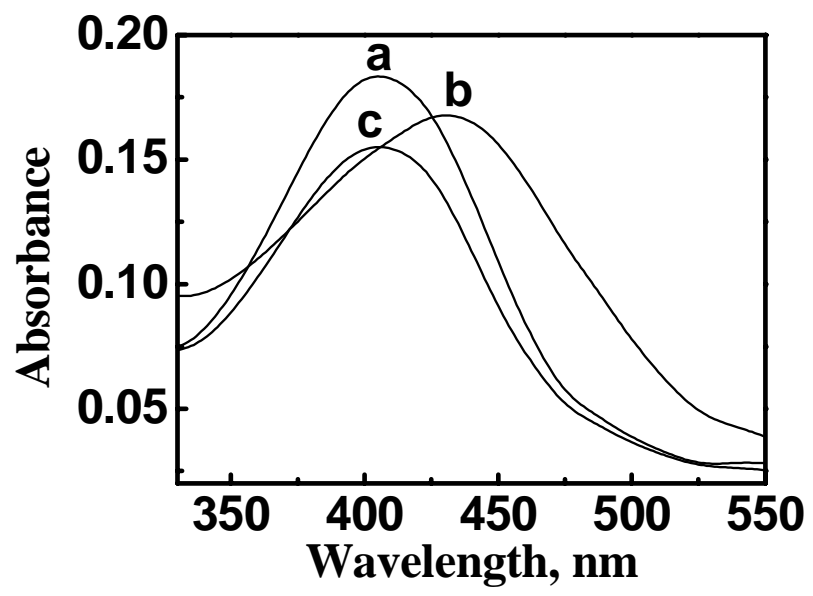

Figure S9. Absorption spectral changes of silver nanoparticles in water (trace a), on addition of $80 \mu \mathrm{M}$ $\mathrm{Pb}^{2+}$ (trace $\mathrm{b}$ ) and after the addition of $95 \mu \mathrm{M}$ EDTA (trace $\mathrm{c}$ ).

10. Absorption Spectral changes of gold nanoparticles in presence of metal ion at pH 6.0
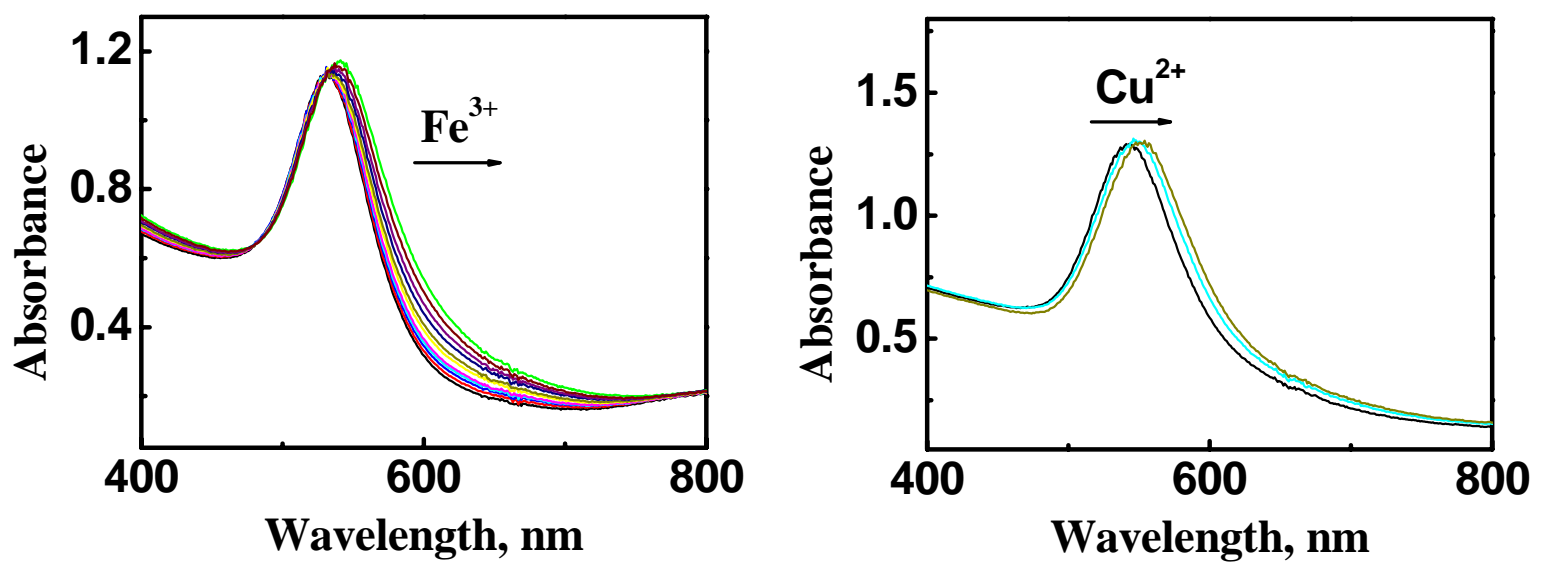

Figure S10. Absorption Spectral changes of gold nanoparticles in water ( $\mathrm{pH} 6.0)$ on addition of (A) $\mathrm{Fe}^{3+}$ (B) $\mathrm{Cu}^{2+}$ ions at $0-100 \mu \mathrm{M}$. 
11. Sensitivity of Au nanoparticles towards $\mathrm{Pb}^{2+}$ ions in presence of other interfering metal cations.

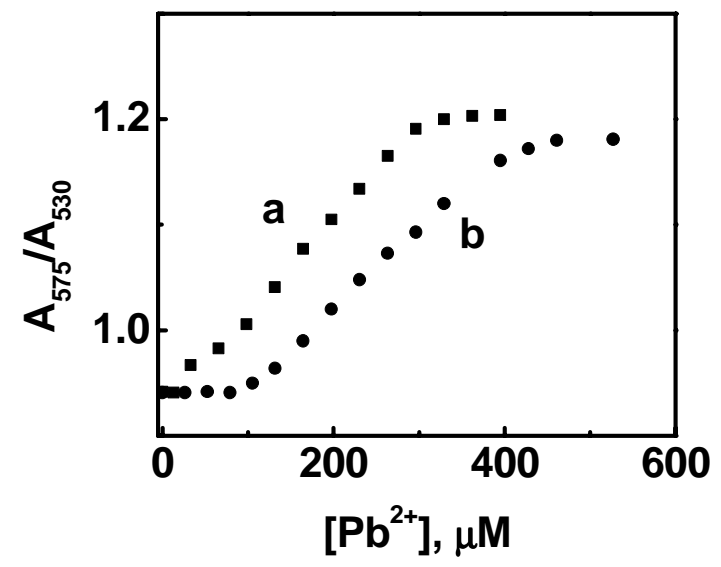

Figure 12. Ratiometric plot showing the sensitivity of nanoparticles towards $\mathrm{Pb} 2+$ ions in presence of different concentrations of interfering metal cations (a) $\left[\mathrm{M}^{\mathrm{n}+}\right]=14 \mu \mathrm{M}$ and (b) $\left[\mathrm{M}^{\mathrm{n}+}\right]=400 \mu \mathrm{M}$.

12. MPWB1K/6-31G(d,p) level optimized structures (Cartesian coordinates in Angstrom unit) and their total energies.
$\mathrm{Au}_{3}{ }^{+}$
$\begin{array}{llll}\text { At. No. } & X & Y & Z\end{array}$
$\begin{array}{llll}79 & 0.000000 & 1.532702 & 0.000000\end{array}$
$79 \quad 1.327478 \quad-0.766355 \quad 0.000000$
$\begin{array}{llll}79 & -1.327478 & -0.766340 & 0.00000\end{array}$

Total Energy $=-405.8698015$ a.u.

Oxidised form of gallic acid (Anion)
At. No. $\mathrm{X}$
$\mathrm{Y}$
Z
$\begin{array}{llll}6 & 0.270454 & -1.191612 & -0.000039\end{array}$
$\begin{array}{llll}6 & -1.065300 & -1.183901 & 0.000131\end{array}$
$\begin{array}{llll}6 & -1.783725 & 0.090889 & 0.000158\end{array}$
$\begin{array}{llll}6 & -0.973211 & 1.399767 & 0.000035\end{array}$
$\begin{array}{llll}6 & 0.459423 & 1.263375 & -0.000096\end{array}$ 


$\begin{array}{lrrr}6 & 1.034371 & 0.046959 & -0.000136 \\ 8 & -2.996244 & 0.094018 & 0.000377 \\ 8 & -1.596620 & 2.438327 & 0.000208 \\ 6 & 2.574390 & -0.088594 & -0.000282 \\ 8 & 2.974013 & -1.263173 & -0.000330 \\ 8 & 3.188644 & 0.981841 & -0.000350 \\ 8 & -1.856659 & -2.269526 & 0.000258 \\ 1 & 0.857402 & -2.096061 & -0.000094 \\ 1 & 1.092018 & 2.136286 & -0.000144 \\ 1 & -2.752913 & -1.913419 & 0.000308\end{array}$

Total Energy $=-644.4241291$ a.u.

\section{Complex1}

\begin{tabular}{lccc} 
At. No. X & Y & Z \\
79 & 0.000000 & 1.539660 & 0.000 \\
79 & -2.653332 & 1.645264 & 0.0000 \\
79 & -1.420956 & -0.707770 & 0.00 \\
6 & 2.782718 & -3.189961 & 0.000 \\
6 & 3.997390 & -3.988881 & 0.000 \\
6 & 5.323542 & -3.220482 & 0.000 \\
6 & 5.284035 & -1.748818 & 0.000 \\
6 & 4.109275 & -1.107825 & 0.000 \\
6 & 2.863077 & -1.849422 & 0.000 \\
8 & 6.387257 & -3.787890 & 0.000 \\
8 & 6.470705 & -1.155024 & 0.000 \\
6 & 1.589344 & -1.049455 & 0.000 \\
8 & 0.523879 & -1.707509 & 0.000 \\
\hline 8 & 1.736772 & 0.195288 & 0.0000 \\
8 & 4.025832 & -5.192153 & 0.000
\end{tabular}




$\begin{array}{llll}1 & 1.830799 & -3.692426 & 0.000 \\ 1 & 4.059127 & -0.032890 & 0.000 \\ 1 & 7.126918 & -1.862318 & 0.000\end{array}$

Total Energy $=-1050.5256472$ a.u.

\section{Complex2}

\begin{tabular}{lccc} 
At. No. $\mathrm{X}$ & $\mathrm{Y}$ & $\mathrm{Z}$ \\
79 & 0.758588 & 6.018837 & -1.582253 \\
79 & 2.792044 & 6.198876 & -3.282339 \\
79 & 2.894521 & 4.471940 & -1.265506 \\
6 & 0.836825 & 2.554837 & 2.739037 \\
6 & 0.358701 & 1.916841 & 3.832783 \\
6 & -1.032879 & 2.209696 & 4.223897 \\
6 & -1.880045 & 3.199863 & 3.410618 \\
6 & -1.240418 & 3.806657 & 2.264921 \\
6 & 0.034050 & 3.482136 & 1.976785 \\
8 & -1.521120 & 1.679670 & 5.205213 \\
8 & -3.009514 & 3.410285 & 3.772797 \\
6 & 0.673585 & 4.117928 & 0.771652 \\
\hline 8 & 1.811751 & 3.687915 & 0.469977 \\
8 & 0.000000 & 5.005005 & 0.196376 \\
8 & 1.014259 & 1.088069 & 4.582702 \\
82 & 0.000000 & 0.000000 & 6.123436 \\
79 & -2.894521 & -4.471940 & -1.26550 \\
79 & -2.792044 & -6.198870 & -3.28233 \\
79 & -0.758588 & -6.018830 & -1.58225 \\
6 & 1.240418 & -3.806657 & 2.264921 \\
6 & 1.880045 & -3.199863 & 3.410618 \\
\hline 6 & & & \\
\hline 6 &
\end{tabular}




\begin{tabular}{lrrr}
6 & 1.032879 & -2.209696 & 4.223897 \\
6 & -0.358701 & -1.916841 & 3.832783 \\
6 & -0.836825 & -2.554837 & 2.739037 \\
6 & -0.034050 & -3.482136 & 1.976785 \\
8 & 1.521120 & -1.679670 & 5.205213 \\
8 & -1.014259 & -1.088069 & 4.582702 \\
6 & -0.673585 & -4.117928 & 0.771652 \\
8 & 0.000000 & -5.005005 & 0.196376 \\
8 & -1.811751 & -3.687915 & 0.469977 \\
8 & 3.009514 & -3.410285 & 3.772797 \\
1 & 1.847526 & 2.378687 & 2.414720 \\
1 & -1.795385 & 4.508156 & 1.667408 \\
\hline 1 & 1.795385 & -4.508156 & 1.667408 \\
\hline 1 & -1.847526 & -2.378687 & 2.414720
\end{tabular}

Total Energy $=-2103.40267192$ a.u.

\section{Complex3}

$\begin{array}{lrcc}\text { At. No. } \mathrm{X} & \mathrm{Y} & \mathrm{Z} \\ 79 & -7.538803 & -6.052851 & -1.10555 \\ 79 & -9.354484 & -6.734800 & 0.69555 \\ 79 & -7.343999 & -5.147583 & 1.44095 \\ 6 & -3.245686 & -2.784582 & 0.038710 \\ 6 & -2.193460 & -1.983806 & -0.246703 \\ 6 & -1.876606 & -1.737437 & -1.666433 \\ 6 & -2.690193 & -2.418638 & -2.763076 \\ 6 & -3.780204 & -3.252549 & -2.325112 \\ 6 & -4.020109 & -3.403846 & -1.004802 \\ 8 & -0.972738 & -0.979468 & -1.956918 \\ 8 & -2.383236 & -2.225637 & -3.918054\end{array}$




\begin{tabular}{|c|c|c|c|}
\hline 6 & -5.202452 & -4.232999 & -0.594447 \\
\hline 8 & -5.578891 & -4.098743 & 0.590285 \\
\hline 8 & -5.695856 & -4.968353 & -1.486282 \\
\hline 8 & -1.428964 & -1.399004 & 0.627160 \\
\hline 82 & 0.028117 & -0.035342 & 0.020162 \\
\hline 79 & -7.271004 & 5.182758 & -1.44810 \\
\hline 9 & -9.265873 & 6.790287 & -0.70102 \\
\hline 79 & -7.448949 & 6.095588 & 1.094341 \\
\hline 6 & -3.742068 & 3.2386540 & 2.324619 \\
\hline 6 & -2.669170 & 2.3860800 & 2.769974 \\
\hline 6 & -1.856237 & 1.6982100 & 1.678869 \\
\hline 6 & -2.159748 & 1.9489190 & 0.259784 \\
\hline 6 & -3.197069 & 2.7651700 & -0.034792 \\
\hline 6 & -3.970496 & 3.3960740 & 1.003190 \\
\hline 8 & -0.962589 & 0.9276760 & 1.975336 \\
\hline 8 & -1.392680 & 1.3557660 & -0.609444 \\
\hline 6 & -5.135650 & 4.2452430 & 0.586559 \\
\hline 8 & -5.618366 & 4.9887250 & 1.477603 \\
\hline 8 & -5.515009 & 4.1131690 & -0.597619 \\
\hline 8 & -2.369819 & 2.1940750 & 3.927323 \\
\hline 79 & 7.470264 & 6.080905 & -1.103796 \\
\hline 79 & 9.317245 & 6.765775 & 0.666711 \\
\hline 79 & 7.298644 & 5.202437 & 1.449484 \\
\hline 6 & 3.2105190 & 2.7976700 & 0.089906 \\
\hline 6 & 2.1715590 & 1.9793230 & -0.196695 \\
\hline 6 & 1.8649590 & 1.7153650 & -1.609317 \\
\hline 6 & 2.6644990 & 2.3928510 & -2.708088 \\
\hline 6 & 3.7372570 & 3.2479040 & -2.274852 \\
\hline 6 & 3.9771270 & 3.4185710 & -0.954877 \\
\hline 8 & 0.9695390 & 0.9312760 & -1.892015 \\
\hline 8 & 2.3585660 & 2.1899030 & $-3.8647^{\prime}$ \\
\hline
\end{tabular}




\begin{tabular}{llll}
6 & 5.1509250 & 4.2588190 & -0.557151 \\
8 & 5.5502750 & 4.1242130 & 0.622169 \\
8 & 5.6240310 & 5.0049680 & -1.452723 \\
8 & 1.4086940 & 1.3844820 & 0.681743 \\
79 & 7.297998 & -5.212242 & -1.45140 \\
79 & 9.334404 & -6.763568 & -0.69081 \\
79 & 7.508985 & -6.0834800 & 1.102563 \\
6 & 3.732877 & -3.310081 & 2.2979990 \\
6 & 2.649835 & -2.468218 & 2.7323750 \\
6 & 1.846850 & -1.794331 & 1.6372090 \\
6 & 2.151677 & -2.055356 & 0.2275260 \\
6 & 3.200329 & -2.860319 & -0.0638940 \\
\hline 6 & 3.975897 & -3.475676 & 0.9773100 \\
\hline 8 & 0.946556 & -1.013554 & 1.9223310 \\
\hline 8 & 1.378683 & -1.469577 & -0.6499000 \\
\hline 6 & 5.159593 & -4.296216 & 0.5738880 \\
8 & 5.652916 & -5.034838 & 1.4651170 \\
\hline 8 & 5.547547 & -4.155058 & -0.6085240 \\
\hline 8 & 2.345133 & -2.269674 & 3.8912340 \\
1 & -3.518331 & -2.967527 & 1.0636050 \\
1 & -4.391054 & -3.744000 & -3.0612650 \\
1 & -4.349520 & 3.740164 & 3.0570520 \\
1 & -3.457723 & 2.954638 & -1.0615630 \\
1 & 3.474872 & 2.9917030 & 1.1149880 \\
\hline 1 & 4.340843 & 3.7420340 & -3.015508 \\
\hline 1.342089 & -3.797653 & 3.0382680 \\
\hline 6 & -3.047745 & -1.0903370
\end{tabular}

Total Energy $=-4203.33851665$ a.u. 
